Andrea L. Vavere, MS, MPH

Armin Arbab-Zadeh, MD, PhD

Carlos E. Rochitte, MD, PhD

Marc Dewey, MD

Hiroyuki Niinuma, MD, PhD

Ilan Gottlieb, MD

Melvin E. Clouse, MD

David E. Bush, MD

John W. M. Hoe, MD

Albert de Roos, MD

Christopher Cox, PhD

João A. C. Lima, MD

Julie M. Miller, MD

\title{
Coronary Artery Stenoses:
}

Accuracy of 64-Detector Row CT

Angiography in Segments with Mild,

Moderate, or Severe Calcification-A

\section{Subanalysis of the CORE-64 Trial ${ }^{1}$}

Materials and Methods:

Results:

${ }^{1}$ From the Department of Cardiology, Johns Hopkins Hospital, 600 N Wolfe St, Carnegie 568, Baltimore, MD 21287 (A.L.V., A.A., I.G., D.E.B., J.A.C.L., J.M.M.); INCOR Heart Institute, University of São Paulo, São Paulo, Brazil (C.E.R.); Department of Radiology, Charité-Universitätsmedizin Berlin, Medical School, Humboldt-Universität und Freie Universität zu Berlin, Berlin, Germany (M.D.); Department of Cardiology, Iwate Medical University, Morioka, Japan (H.N.); Department of Radiology, Beth Israel Deaconess Medical Center, Harvard University, Boston, Mass (M.E.C.); MediRad Associates, CT Centre, Mt Elizabeth Hospital, Singapore (J.W.M.H.); Department of Radiology, Leiden University Medical Center, Leiden, the Netherlands (A.d.R.); and Department of Epidemiology, Johns Hopkins Bloomberg School of Public Health, Baltimore, Md (C.C.). Received March 14, 2011; revision requested April 19; revision received May 13; accepted May 20; final version accepted May 27. Supported by Toshiba Medical Systems, Doris Duke Charitable Foundation, and Donald W. Reynolds Foundation. Address correspondence to J.M.M. (e-mail: jmmiller@jhmi.edu).

( RSNA, 2011

Conclusion:
Purpose:

To evaluate the influence of cross-sectional arc calcification on the diagnostic accuracy of computed tomography (CT) angiography compared with conventional coronary angiography for the detection of obstructive coronary artery disease (CAD).

Institutional Review Board approval and written informed consent were obtained from all centers and participants for this HIPAA-compliant study. Overall, 4511 segments from 371 symptomatic patients (279 men, 92 women; median age, 61 years [interquartile range, 53-67 years]) with clinical suspicion of CAD from the CORE-64 multicenter study were included in the analysis. Two independent blinded observers evaluated the percentage of diameter stenosis and the circumferential extent of calcium (arc calcium). The accuracy of quantitative multidetector CT angiography to depict substantial $(\geq 50 \%)$ stenoses was assessed by using quantitative coronary angiography (QCA). Cross-sectional arc calcium was rated on a segment level as follows: noncalcified or mild $\left(<90^{\circ}\right)$, moderate $\left(90^{\circ}-180^{\circ}\right)$, or severe $\left(>180^{\circ}\right)$ calcification. Univariable and multivariable logistic regression, receiver operation characteristic curve, and clustering methods were used for statistical analyses.

A total of 1099 segments had mild calcification, 503 had moderate calcification, 338 had severe calcification, and 2571 segments were noncalcified. Calcified segments were highly associated $(P<.001)$ with disagreement between CTA and QCA in multivariable analysis after controlling for sex, age, heart rate, and image quality. The prevalence of CAD was $5.4 \%$ in noncalcified segments, $15.0 \%$ in mildly calcified segments, $27.0 \%$ in moderately calcified segments, and $43.0 \%$ in severely calcified segments. A significant difference was found in area under the receiver operating characteristic curves (noncalcified: 0.86 , mildly calcified: 0.85 , moderately calcified: 0.82 , severely calcified: $0.81 ; P<.05$ ).

In a symptomatic patient population, segment-based coronary artery calcification significantly decreased agreement between multidetector CT angiography and QCA to detect a coronary stenosis of at least $50 \%$.

${ }^{\circ}$ RSNA, 2011

Supplemental material: http://radiology.rsna.org/lookup /suppl/doi:10.1148/radiol.11110537/-/DC1 
C oronary artery disease (CAD) remains the leading cause of death in the United States for both men and women (1). Multidetector computed tomographic (CT) coronary angiography for the noninvasive detection of atherosclerosis and obstructive CAD has been used in a variety of patient populations suspected of having CAD owing to its high diagnostic accuracy, as shown in previous single and multicenter studies in selected populations with good multidetector CT angiography image quality (2-9). Despite technical advances improving both spatial and temporal resolutions (10-12), coronary artery calcification continues to degrade image quality and limits the diagnostic accuracy of multidetector CT angiography as compared with conventional coronary angiography (CCA) for the detection of obstructive CAD. Researchers in several studies (7,13-15) have reported that multidetector CT angiography in patients with severe coronary artery calcification (Agatston calcium score $>400$ ) remains less effective as a diagnostic tool than in patients with mild, moderate, or no coronary artery calcification. In a previous patient-based analysis, we reported that

\section{Advances in Knowledge}

- The prevalence of coronary artery disease (CAD) in noncalcified segments is substantially lower than in segments with mild, moderate, or severe calcification.

- Cross-sectional arc calcification is a strong predictor of diagnostic disagreement between CT angiography and conventional angiography after controlling for sex, age, heart rate, and image quality.

- Diagnostic accuracy by area under the receiver operating characteristic curve is significantly lower by degree of segment calcification; the positive likelihood ratio in a coronary segment with disease is acceptable in noncalcified segments, but it is lower than is acceptable in segments with moderate or severe calcification. overall coronary artery calcification did not reduce the diagnostic accuracy of multidetector CT angiography compared with CCA in a cohort of patients with an Agatston coronary calcium score of 600 or less; however, in vessel-based analysis, the diagnostic capability was reduced in those patients with a higher Agatston score (8). The aim of the our study was to further evaluate the influence of coronary artery calcification at the individual segment level on the diagnostic accuracy of multidetector CT angiography in comparison with CCA.

\section{Materials and Methods}

Financial grant support was received from Toshiba Medical Systems (Nasu, Japan); the Doris Duke Charitable Foundation (New York, NY); the National Heart, Lung, and Blood Institute (grants RO1-HL66075-01 and HO1-HC95162-01); the National Institute on Aging (grant RO1-AG021570-01); and the Donald W. Reynolds Foundation (Las Vegas, Nev). The multidetector CT contrast agent was provided by Bracco Diagnostics (Princeton, NJ). The authors had complete control of the data and the information submitted for publication.

\section{Study Population}

The Coronary Evaluation Using Multidetector Spiral Computed Tomography Angiography Using 64 Detectors, or CORE64, trial, a multicenter international trial, prospectively enrolled 371 symptomatic patients (279 men, 92 women; median age, 61 years [interquartile range $\{\mathrm{IQR}\}, 53-$ 67 years]) suspected of having CAD who were at least 40 years old and were referred for CCA into a single-blinded cohort study conducted at nine centers in seven countries $(6,16)$ (Appendix E1 [online]).

\section{Implication for Patient Care}

- The clinical utility of multidetector CT angiography to identify a patient with obstructive CAD has been shown; however, its utility for determining the presence of substantial CAD in a segment is limited by the presence and extent of coronary calcification.
The complete CORE-64 protocol and methods have been previously described $(6,16)$. Inclusion criteria were symptomatic patients who were at least 40 years old, were scheduled for CCA, and were willing to provide written informed consent. Participant exclusion criteria were a history of allergic reaction to iodinated contrast agents, renal failure, multiple myeloma, previous organ transplant, elevated serum creatinine $(>1.5 \mathrm{mg} / \mathrm{dL}$ or calculated creatinine clearance $<60 \mathrm{~mL}$ ), atrial fibrillation, tachyarrhythmia, advanced atrioventricular block, evidence of severe symptomatic heart failure (New York Heart Association class III or IV), severe aortic stenoses, previous coronary artery bypass or other cardiac surgery, coronary artery intervention within the last 6 months, contraindication to $\beta$-blockers, body mass index higher than $40 \mathrm{~kg} / \mathrm{m}^{2}$, and presence or history of any condition that the investigator judged to be a relevant reason for exclusion. The Health Insurance Portability and Accountability Act-compliant study protocol

Published online before print

10.1148/radiol.11110537 Content codes: CA CT

Radiology 2011; 261:100-108

Abbreviations:

$\mathrm{AUC}=$ area under receiver operating characteristic curve $\mathrm{CAD}=$ coronary artery disease

$\mathrm{CCA}=$ conventional coronary angiography

$\mathrm{Cl}=$ confidence interval

$\mathrm{IQR}=$ interquartile range

$\mathrm{QCA}=$ quantitative coronary angiography

\section{Author contributions:}

Guarantors of integrity of entire study, A.L.V., H.N., M.E.C J.A.C.L., J.M.M.; study concepts/study design or data acquisition or data analysis/interpretation, all authors; manuscript drafting or manuscript revision for important intellectual content, all authors; approval of final version of submitted manuscript, all authors; literature research, A.L.V., A.A., J.A.C.L., J.M.M.; clinical studies, A.A., M.D., H.N., M.E.C., J.W.M.H., C.C., J.A.C.L., J.M.M.; statistical analysis, A.L.V., H.N., C.C., J.M.M.; and manuscript editing, A.L.V., A.A., C.E.R., M.D., H.N., I.G., M.E.C., D.E.B., A.d.R., C.C., J.A.C.L., J.M.M.

\section{Funding:}

This research was supported by the National Heart, Lung, and Blood Institute (grants R01-HL66075-01 and H01-HC95162-01) and the National Institute on Aging (R01-AG021570-01).

Potential conflicts of interest are listed at the end of this article. 
and informed consent form were approved by the institutional review board at each center as well as a centralized institutional review board at Johns Hopkins Hospital.

\section{Multidetector CT Angiography Acquisition Protocol}

Calcium scanning was performed by using prospective electrocardiographic gating at $120 \mathrm{kV}$ and $300 \mathrm{~mA}$, with gantry rotation of 0.4 second and detector collimation of $4 \times 3.0 \mathrm{~mm}$. For the multidetector CT angiography, retrospective electrocardiographic gating was used with gantry rotation times of 350-400 msec automatically selected to allow synchrony with the heartbeat to enable efficient adaptive multisegment image reconstruction (17). On the basis of body mass index, an acquisition tube current of 240-400 mAs was used with an unchanging tube voltage of $120 \mathrm{kV}$ to meet a predetermined gender-specific effective radiation dose of approximately 12-15 mSv, not to exceed $20 \mathrm{mSv}$. Iopamidol (Isovue 370; Bracco Diagnostics) was administered intravenously with image acquisition triggered automatically at $180 \mathrm{HU}$ in the descending aorta. Images were acquired from the carina through the cardiac apex during one breath hold. All images were acquired by using $64 \times$ 0.5-mm high-detection CT angiography (Toshiba Medical Systems).

$\beta$-Blockers were given when the resting heart rate was above 70 beats per minute to reduce the heart rate to below 65 beats per minute. If the mean heart rate could not be reduced to below 80 beats per minute prior to or during image acquisition, the patient was prespecified to be excluded from the primary study analysis. Sublingual short-acting nitroglycerin was routinely given if systolic blood pressure was above $110 \mathrm{~mm} \mathrm{Hg}$. All multidetector CT studies were performed 30 or fewer days before CCA.

\section{Multidetector CT Angiography Data Management and Analysis}

Raw image data from all multidetector CT angiography scans were transferred to a centralized core laboratory where all images were reconstructed. Images were reconstructed at $0.5-\mathrm{mm}$ section thickness with overlap of $0.3 \mathrm{~mm}$. ImageXact software (Toshiba Medical Systems) was used to select the systolic and diastolic phases with the least cardiac motion by using both standard (FC43) and hard (FC05) convolution kernels. A temporal window of plus or minus 20 msec was used to optimize image reconstruction on per-patient and per-vessel bases for both systolic and diastolic reconstructions. Electrocardiographic editing was used to exclude arrhythmias and decrease cardiac motion.

The CT Core Imaging Laboratory at Johns Hopkins University reconstructed images in multiple cardiac phases for interpretation by two independent cardiologists (A.A. and I.G) with 5 or more years of prior cardiac imaging experience who were blinded to any patient information and used independent workstations with dedicated cardiac multidetector CT analysis software (Vitrea 2, version 3.9.0.1; Vital Images, Minnetonka, Minn). Images were first visually assessed by using a 19-segment coronary model (16). Quantitative assessment of the degree of diameter stenosis was then performed in crosssectional and longitudinal projections in segments visually identified as having $30 \%$ or greater stenosis. Measurements were made by using a semiautomatic contour-detection algorithm, electronic calipers, and rulers in crosssectional and longitudinal projections. Final determinations of reference and lumen diameters were made only after manual contour editing (16). Segments with substantial discrepancies underwent a consensus process that incorporated a third cardiologist with more than 30 years of cardiac imaging experience. In segments in which the difference between the two principal readers was not substantial, a consensus score was derived through averaging. A substantial difference was defined as the maximum percentage diameter stenosis crossing the $50 \%$ or $70 \%$ thresholds. Additionally, if any one reader judged a segment to be nonassessable, the segment underwent a consensus interpretation. Segments were deemed nonassessable only if there were no quantitative measurements made by any of the readers.
The extent of segment calcification was measured in all segments of the coronary tree by using an adapted crosssectional arc calcification method (18). In brief, coronary artery segments were visually examined in cross section by the two independent observers who were blinded to patient information, and the greatest circumferential extent of calcium within the coronary segment (arc calcification) was independently assessed in degrees according to the following ordinal scale: 1 = noncalcified segments, 2 = mild calcification (cross-sectional arc calcium $<90^{\circ}$ ), $3=$ moderate calcification (cross-sectional arc calcium of $90^{\circ}-180^{\circ}$ ), and $4=$ severe calcification $\left(\right.$ cross-sectional arc calcium $>180^{\circ}$ ) (Fig 1). Similarly, segment image quality was evaluated by using an ordinal scale: 0 = unevalulable, 1 = poor quality, 2 = adequate quality, and $3=\operatorname{good}$ or optimal quality. The final consensus segment calcium burden and image quality scores were derived through averaging.

\section{CCA Data Acquisition and Analysis}

CCA was performed by using standard angiographic techniques within 30 days following multidetector CT angiography. Intracoronary nitroglycerine was administered $(150-200 \mu \mathrm{g})$ prior to acquisition of the first image of the left and right coronary arteries to standardize coronary tone. Coronary angiographic images were saved in Digital Imaging and Communications in Medicine format and forwarded to the Angiographic Core Laboratory at Johns Hopkins University for analysis.

Quantitative coronary angiography (QCA) was performed by using an edgedetection algorithm with validated commercially available software (CAAS II QCA Research, version 2.0.1; PIE Medical Imaging, Maastricht, the Netherlands). With the outer diameter of the contrast agent-filled catheter as the calibration standard, the minimum lumen diameter in diastole or near the end of the diastolic phase was measured from orthogonal projections. The most substantial stenoses within each coronary segment were analyzed with quantitative assessment of the degree of stenosis. The percentage of stenosis was derived from 
the view with the greatest reduction of diameter (the worst view) with the least foreshortening of the segment in question. The reference diameter is an interpolated diameter of normal segments proximal and distal to the lesion. Segments that could not be accurately visualized owing to reduced image quality were excluded. Segmental disease was analyzed in each vessel by using the 19-segment model used with the multidetector CT angiography. Lesions causing a $50 \%$ or greater reduction of the lumen were considered obstructive.

\section{Cross-Modality Segment Adjudication Process}

Following analysis completion, all stenosis measurements were locked, and the adjudication was performed to ensure that segment scores for both multidetector CT angiography and QCA were derived from the same segments. The adjudication methods have been previously described $(6,16)$; briefly, all segments for every patient with at least one lesion with $50 \%$ or greater obstruction as determined with either QCA or multidetector CT angiography were adjudicated by a committee. No visual or quantitative measurements with either modality could be modified during the adjudication process; only the label for alignment of the attributed segment could be altered.

\section{Statistical Analysis}

Statistical analyses were performed with Stata statistical software (release 10.0; StataCorp, College Station, Tex). Univariate and multivariable logistic regression analyses were performed to compute the odds ratios for disagreement between multidetector CT angiography and QCA according to the degree of segment calcification. Computation of confidence limits for segments took into account within-patient clustering by using logistic regression with generalized estimating equations (19). Sensitivity, specificity, and positive and negative predictive values were calculated by standard methods (20). The receiver operating characteristic curve was used as the measure of diagnostic accuracy. Computation of confidence limits for the area under the receiver operating characteristic curve
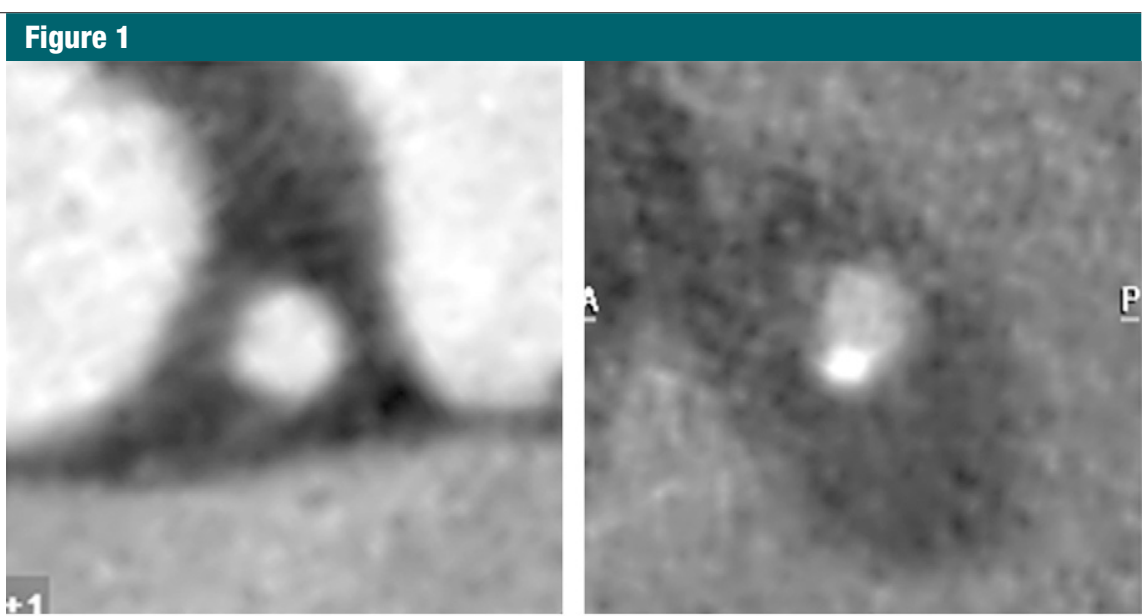

a.

b.
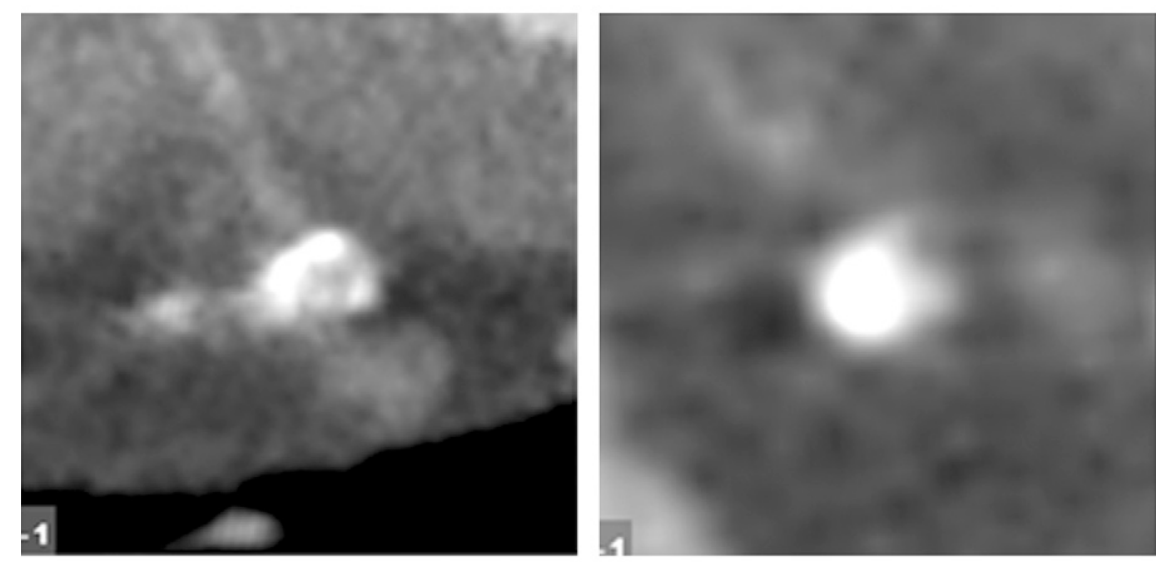

c.

d.

Figure 1: CT images show (a) noncalcified, (b) mildly calcified (cross-sectional arc calcium $<90^{\circ}$ ), (c) moderately calcified (cross-sectional arc calcium 90 $-180^{\circ}$ ), and (d) severely calcified (cross-sectional arc calcium $>180^{\circ}$ ) segments. Segment calcification was measured by using the cross-sectional arc method (19).

(AUC) took account of within-patient clustering by means of bootstrap resampling using the percentile method with 2000 bootstrap samples (21). The reference standard for receiver operating characteristic curve analysis was 50\% or greater diameter stenosis at QCA. AUCs for different groups were compared by using bootstrap confidence intervals (CIs) for the difference between the AUCs. All tests were two-tailed, the significance threshold was a $P$ value less than .05 , and CIs were $95 \%$.

\section{Results}

\section{Baseline Demographics}

Of 405 patients who gave consent, 34 were excluded because of major protocol deviations, resulting in a final study population of 371 patients. Of the 371 patients (279 men, 92 women), 291 had Agatston scores below 600. Baseline demographics are shown in Table 1 . The median age was 61 years (IQR, 53-67 years). The median body mass index was $27 \mathrm{~kg} / \mathrm{m}^{2}$ (IQR, 25-30 kg/m²); median heart rate, 61 beats per minute (IQR, 55-69 beats per minute); and median Agatston score, 148 (IQR, 8-478). Seventy percent (260 of 371) of patients had a history of hypertension; $64 \%$ (236 of 371), dyslipidemia; 26\% (97 of 371 ), diabetes; $18 \%$ (67 of 371$)$, current smokers; $42 \%$ (154 of 371 ), former smokers; 40\% (150 of 371), never smokers; and 30\% (111 of 371), CAD. One hundred eighty-five subjects $(50 \%)$ received $\beta$-blockers, and 356 (96\%) 


\section{Table 1}

\section{Patient Characteristics}

CT Angiography Quality

\begin{tabular}{|c|c|c|c|c|}
\hline \multirow[b]{2}{*}{ Parameter } & \multirow[b]{2}{*}{ All Patients $(n=371)$} & & & \\
\hline & & Good $(n=142)$ & Adequate $(n=189)$ & Poor $(n=40)$ \\
\hline \multicolumn{5}{|l|}{ Patient demographics } \\
\hline \multicolumn{5}{|l|}{ Sex* } \\
\hline Male & $279(75)$ & $111(78)$ & $141(75)$ & $27(68)$ \\
\hline Female & $92(25)$ & $31(22)$ & $48(25)$ & $13(33)$ \\
\hline Age $(y)^{\dagger}$ & $61(53-67)$ & $59(53-66)$ & $61(53-67)$ & $62(52-70)$ \\
\hline \multicolumn{5}{|l|}{ Ethnicity* } \\
\hline White & $260(70)$ & $83(58)$ & $146(77)$ & $31(78)$ \\
\hline Asian & $78(21)$ & $50(35)$ & $26(14)$ & $2(5)$ \\
\hline Black & $20(5)$ & $4(3)$ & $10(5)$ & $6(15)$ \\
\hline Other & $13(4)$ & $5(4)$ & $7(4)$ & $1(2)$ \\
\hline Body mass index $\left(\mathrm{kg} / \mathrm{m}^{2}\right)^{\dagger}$ & $27(25-30)$ & $26(24-29)$ & $28(25-31)$ & $30(27-33)$ \\
\hline \multicolumn{5}{|l|}{$\begin{array}{c}\text { Multidetector CT angiography } \\
\text { characteristics }\end{array}$} \\
\hline Agatston score $^{\dagger}$ & $148(8-478)$ & $125(1-338)$ & $200(26-689)$ & $145(11-573)$ \\
\hline Heart rate $(\text { beats } / \mathrm{min})^{\dagger}$ & $61(55-69)$ & 57 (52-62) & $60(55-65)$ & $65(58-75)$ \\
\hline $\begin{array}{l}\text { Heart rate variability } \\
\quad \text { (beats per scan) }^{\dagger}\end{array}$ & $3(2-5)$ & $2(1-4)$ & $3(2-5)$ & $4(3-9)$ \\
\hline Breath artifact ${ }^{\ddagger}$ & 24 & 0 & 15 & 9 \\
\hline Ectopy ${ }^{\ddagger}$ & 21 & 2 & 14 & 5 \\
\hline
\end{tabular}

* Data are numbers of patients, with percentages in parentheses.

${ }^{\dagger}$ Data are medians, with IQRs in parentheses.

$\ddagger$ Data are numbers of patients.

received nitroglycerin before CT data acquisition.

\section{Diagnostic Accuracy by Level of Coronary Arc Calcification}

A total of 4511 segments, from 371 patients in which segment calcium burden measurements were made, were included in the analysis. Good agreement was observed between the readers in the assessment of segment calcium burden $(\kappa=$ 0.65). A total of 2571 (57\%) were free of coronary calcification, and 1940 (43\%) had some degree of calcification. Of the noncalcified segments, 139 (5\%) had obstructive noncalcified plaques. Of all segments, 1099 (24\%) had mild calcification, 503 (11\%) had moderate calcification, and 338 (7\%) had severe calcification. In segments with coronary calcium, the prevalence of obstructive disease was $15 \%$ for mild calcification, $27 \%$ for moderate calcification, and $43 \%$ for severe calcification. As the degree of coronary calcification increased, the sensitivity of multidetector CT angiography increased, ranging from 44\% (61 of 139) in noncalcified segments to $81 \%$ (118 of 145) in segments with severe calcification $(P=$ .014). However, as the degree of calcification increased, the specificity of multidetector CT angiography decreased, ranging from 99\% (2401 of 2432) in noncalcified segments to $71 \%$ (137 of 193) in segments with severe calcification $(P=.010$, Table 2$)$. Positive predictive values were similar between groups, while negative predictive values were dissimilar, with noncalcified segments having the highest negative predictive value. Noncalcified segments had a positive likelihood ratio of 34.4 (95\% CI: 23.1, $51.2)$ as compared with 9.9 (95\% CI: 7.5, 13.1), 4.3 (95\% CI: 3.3, 5.5), and 2.8 (95\% CI: 2.2, 3.5) for mild, moderate, and severe segment calcium, respectively. The overall diagnostic power of multidetector CT angiography was evaluated by computing the AUC. The AUC ranged from 0.86 (95\% CI: $0.82,0.90)$ for noncalcified segments to 0.81 (95\% CI: $0.76,0.85)$ for severely calcified segments (Fig 2). A significant difference in AUC was found between noncalcified segments and segments with varying magnitude of calcification $(P<.05)$. The difference in AUC was 0.01 (95\% CI: 0.01, 0.01) between noncalcified and mildly calcified segments, 0.04 (95\% CI: $0.04,0.04)$ between noncalcified and moderately calcified segments, 0.06 (95\% CI: 0.05, 0.06) between noncalcified and severely calcified segments, 0.03 (95\% CI: $0.03,0.03)$ between mildly and moderately calcified segments, 0.04 (95\% CI: $0.04,0.05)$ between mildly and severely calcified segments, and 0.02 (95\% CI: $0.02,0.02)$ between moderately and severely calcified segments.

Among the segments with falsepositive findings at multidetector CT angiography, $85 \%$ (173 of 204) had some degree of calcification (mild: $28 \%$ [57 of 204], moderate: $29 \%$ [60 of 204], severe: $27 \%$ [56 of 204]); while, of the segments with false-negative findings, $37 \%$ (78 of 211) were noncalcified, and $63 \%$ (133 of 211) had some calcification (mild: 31\% [65 of 211], moderate: 19\% [41 of 211], severe: 13\% [27 of 211]). Among segments with true-positive findings, $24 \%$ (139 of 585$)$ were noncalcified, and 76\% (446 of 585) had some degree of calcification. However, among the segments with true-negative findings, $5 \%$ (193 of 3926) had severe calcification, 9\% (365 of 3926) had moderate calcification, 24\% (936 of 3926) had mild calcification, and $62 \%$ (2432 of 3926 ) were noncalcified. The influence of segment calcification on the percentage of segments that were concordant, overestimated (false-positive), or underestimated (false-negative) with multidetector CT angiography compared with CCA is presented in Figure 3.

The influence of calcification on segment-level image quality is presented in Figure 4. Eighty-six percent (2204 of 2571) of the noncalcified segments were of good-to-adequate image quality, while $54 \%$ (433 of 800 ) of the segments with poor image quality had some degree of calcification.

\section{Multivariable Regression}

In multivariable regression analysis, segments with calcification were highly 


\section{Table 2}

\section{The Effect of Segment Calcification on the Diagnostic Accuracy of Multidetector CT Angiography versus CCA}

\begin{tabular}{lllll} 
Parameter & Noncalcified $(n=2571)$ & Mild Calcium $(n=1099)$ & Moderate Calcium $(n=503)$ & Severe Calcium $(n=338)$ \\
\hline Prevalence (\%) & $5.4(139 / 2571)$ & $15.0(163 / 1099)$ & $27.0(138 / 503)$ & $43.0(145 / 338)$ \\
Sensitivity (\%) & $43.9(61 / 139)[35.5,52.5]$ & $60.1(98 / 163)[52.2,67.7]$ & $70.3(97 / 138)[61.9,77.8]$ & $81.4(118 / 145)[74.1,87.4]$ \\
Specificity (\%) & $98.7(2401 / 2432)[98.2,99.1]$ & $93.9(879 / 936)[92.2,95.4]$ & $83.6(305 / 365)[79.4,87.2]$ & $71.0(137 / 193)[64.0,77.3]$ \\
Positive predictive value (\%) & $66.3(61 / 92)[55.7,75.8]$ & $63.2(98 / 155)[55.1,70.8]$ & $61.8(97 / 157)[53.7,69.4]$ & $67.8(118 / 174)[60.3,74.7]$ \\
Negative predictive value (\%) & $96.9(2401 / 2479)[96.1,97.5]$ & $93.1(879 / 944)[91.3,94.6]$ & $88.2(305 / 346)[84.3,91.4]$ & $83.5(137 / 164)[77.0,88.9]$ \\
AUC & $0.86[0.82,0.90]$ & $0.85[0.81,0.89]$ & $0.82[0.78,0.87]$ & $0.81[0.76,0.85]$ \\
Positive likelihood ratio & $34.4[23.1,51.2]$ & $9.9[7.5,13.1]$ & $4.3[3.3,5.5]$ & $2.8[2.2,3.5]$ \\
Negative likelihood ratio & $0.57[0.49,0.66]$ & $0.43[0.35,0.51]$ & $0.36[0.27,0.46]$ & $0.26[0.18,0.37]$
\end{tabular}

Note.-Data in parentheses were used to calculate percentages, and data in brackets are $95 \% \mathrm{Cls}$.

associated with diagnostic disagreement between multidetector CT angiography and QCA $(P<.001)$ after adjusting for sex, age, image quality, heart rate during acquisition, and the interaction of age and coronary calcium (Table 3 ). The odds ratio for disagreement in mildly calcified plaques as compared with noncalcified plaques was 2.57 (95\% CI: 1.91, $3.44)$; in moderately calcified plaques, 4.14 (95\% CI: 2.90, 5.91); and in severely calcified plaques, 3.94 (95\% CI: $2.64,5.88$ ) (Table 3). Men had a higher odds ratio for disagreement compared with women (1.86 [95\% CI: 1.36, 2.55]), and segments with poor or adequate quality had higher odds ratios for disagreement (3.52 [95\% CI: 2.40, 5.17] and 4.80 [95\% CI: 3.13, 7.34], respectively) relative to segments with optimal quality (Table 3).

\section{Discussion}

Multidetector CT angiography is a promising test for the noninvasive detection of coronary artery stenosis in patients because of its noninvasive nature, high sensitivity, and negative predictive value, as found in several previous studies using 16,32 , or 64 detector rows (2-9). Researchers in several studies $(7,13-15)$ have reported that multidetector CT angiography in patients with severe coronary calcification (Agatston score $>400$ ) remains less effective than that in patients with mild, moderate, or no coronary calcification. In our study, coronary artery calcification was associated with reduced diagnostic accuracy as as-

\section{Figure 2}

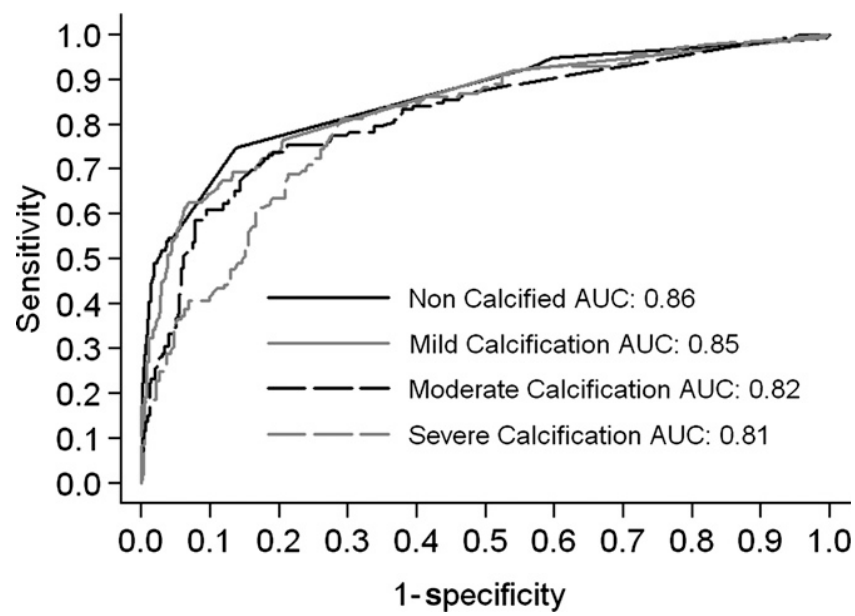

Figure 2: Receiver operating characteristic curves and corresponding AUCs show diagnostic performance of 64-detector row CT angiography for identification of $50 \%$ or greater coronary stenoses at a segment level with QCA as the reference standard.

sessed with the AUC, specificity, and negative predictive value in a segmentbased analysis. The prevalence of disease in the noncalcified group was 5\% as compared with $43 \%$ in the severe calcium group. Since positive and negative predictive values are influenced by the prevalence of disease, positive and negative likelihood ratios have been proposed as better measurements of diagnostic accuracy. Positive likelihood ratios greater than 10 and negative likelihood ratios smaller than 0.1 have been proposed as markers of a given test's satisfactory diagnostic performance. When comparing noncalcified segments to mildly, moderately, or severely calcified segments, noncalcified segments had a positive likelihood ratio of 34.4 (95\% CI: $23.1,51.2)$ as compared with $9.9(95 \%$ CI: 7.5, 13.1), 4.3 (95\% CI: 3.3, 5.5), and 2.8 (95\% CI: 2.2, 3.5), respectively, emphasizing the influence of calcium on the ability of multidetector CT angiography to depict a truly positive segment in the presence of calcium. The negative likelihood ratios were 0.57 (95\% CI: $0.49,0.66)$ for noncalcified segments and 0.43 (95\% CI: $0.35,0.51), 0.36$ (95\% CI: 0.27, 0.46), and 0.26 (95\% CI: $0.18,0.37$ ) for mildly, moderately, and severely calcified segments, respectively. The adjusted odds of a disagreement between multidetector CT angiography 
Figure 3

\section{Concordance Overestimation Underestimation}

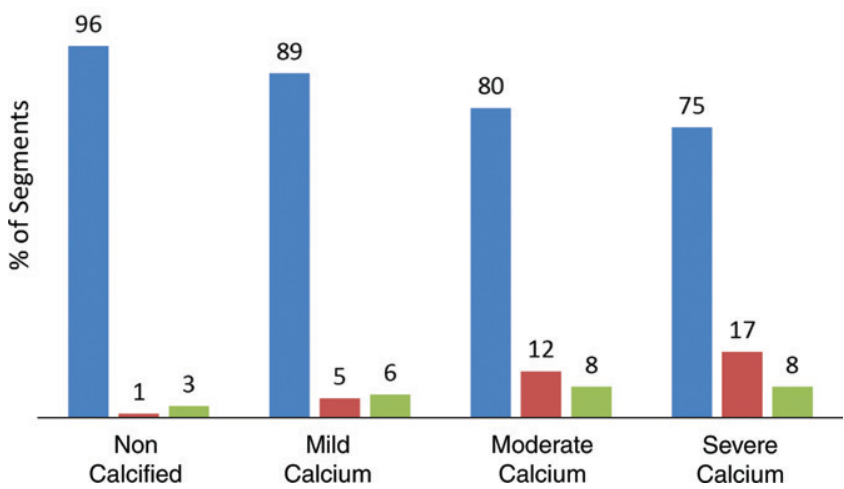

Figure 3: Bar graph shows influence of segment calcification on the concordance, overestimation, and underestimation with multidetector CT angiography compared with CCA. Of the 2571 noncalcified segments, 2462 were concordant, 31 were false-positive, and 78 were false-negative. Of the 1099 mildly calcified segments, 977 were concordant, 57 were false-positive, and 65 were false-negative. Of the 503 moderately calcified segments, 402 were concordant, 60 were false-positive, and 41 were false-negative. Of the 338 severely calcified segments, 255 were concordant, 56 were false-positive, and 27 were false-negative.

\section{Figure 4}

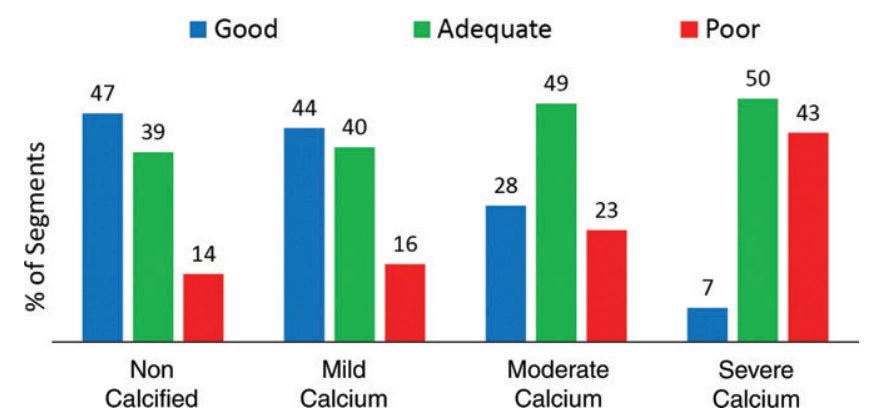

Figure 4: Bar graph shows influence of calcification on segment-level CT image quality. Of the 2571 noncalcified segments, 1208 were of good quality, 996 were of adequate quality, and 367 were of poor quality. Of the 1099 mildly calcified segments, 489 were of good quality, 436 were of adequate quality, and 174 were of poor quality. Of the 503 moderately calcified segments, 142 were of good quality, 247 were of adequate quality, and 114 were of poor quality. Of the 338 severely calcified segments, 25 were of good quality, 168 were of adequate quality, and 145 were of poor quality.

and CCA in mildly calcified segments was 2.57 , that in moderately calcified segments was 4.14 , and that in severely calcified segments was 3.94. Therefore, as the extent of calcium plaque increased, the odds of a misdiagnosis increased.

In a previous subanalysis of the CORE-64 trial (8), coronary calcium as quantified by Agatston score was not associated with reduced multidetector CT angiography diagnostic accuracy compared with CCA in patient-based analyses; however, in vessel-based analyses, coronary calcium was associated with reduced multidetector CT angiography diagnostic accuracy. Furthermore, in a prior study (7) evaluating the effect of coronary calcification on the diagnostic accuracy of multidetector CT angiography compared with CCA in segment-based analyses, the investigators reported sensitivity, specificity, and positive and negative predictive values of $77 \%, 98 \%, 66 \%$, and $99 \%$, respectively, in noncalcified segments; $87 \%$, $93 \%, 72 \%$, and $97 \%$, respectively, in mildly calcified segments; $92 \%, 83 \%$, $65 \%$, and $97 \%$, respectively, in moderately calcified segments; and 93\%, $72 \%, 64 \%$, and $95 \%$, respectively, in severely calcified segments. We found a similar trend from noncalcified to calcified segments, with uniformly lower sensitivities and negative predictive values and similar specificities and positive predictive values.

Previous studies $(7,13,22)$ have found that multidetector CT angiography overestimates the degree of obstructive CAD in the presence of coronary calcification. In our study, $85 \%$ of the segments with false-positive findings had coronary calcification (28\% mild, 29\% moderate, and $27 \%$ severe); however, $76 \%$ of the segments with true-positive findings also had coronary calcification. In severely calcified segments, both overestimation and underestimation occurred. Results of previous studies $(7,22)$ have shown low false-negative rates even in the presence of calcified coronary plaques. We observed similar findings in our study, with $3 \%$ of the noncalcified, $6 \%$ of the mildly calcified, $8 \%$ of the moderately calcified, and $8 \%$ of the severely calcified segments reported as false-negatives. Zhang et a (22) reported that larger calcified coronary artery plaques have higher prevalence rates of obstructive CAD; however, a majority of calcified plaques undergo remodeling and do not lead to luminal narrowing. Similar findings were observed in our study, in which $43 \%$ of the severely calcified, $27 \%$ of the moderately calcified, and $15 \%$ of the mildly calcified segments had obstructive CAD.

In our study and in previous reports, heavy coronary artery calcification was associated with a reduction in image quality and diagnostic accuracy when compared with CCA (2). The interpretation of multidetector CT angiography in the presence of coronary artery calcification is limited by blooming and beamhardening artifacts $(22,23)$, which make it difficult to clearly delineate between 
Table 3

\section{Crude and Adjusted Odds Ratios for Diagnostic Disagreement between Multidetector} CT Angiography and QCA

\begin{tabular}{|c|c|c|c|c|}
\hline \multirow[b]{2}{*}{ Parameter } & \multicolumn{2}{|c|}{ Crude } & \multicolumn{2}{|c|}{ Adjusted $^{*}$} \\
\hline & Odds Ratio & $P$ Value & Odds Ratio & $P$ Value \\
\hline Male vs female sex & $1.93(1.36,2.73)$ & $<.001$ & $1.86(1.36,2.55)$ & $<.001$ \\
\hline Age & $1.02(1.00,1.03)$ & .03 & $1.01(0.99,1.02)$ & .11 \\
\hline \multicolumn{5}{|l|}{ Arc calcium $^{\dagger}$} \\
\hline None $^{\ddagger}$ & 1.0 & $<.001$ & 1.0 & $<.001$ \\
\hline Mild & $2.82(2.12,3.75)$ & & $2.57(1.91,3.44)$ & \\
\hline Moderate & $5.67(4.09,7.86)$ & & $4.14(2.90,5.91)$ & \\
\hline Severe & $7.35(5.13,10.53)$ & & $3.94(2.64,5.88)$ & \\
\hline \multicolumn{5}{|l|}{ Segment image quality } \\
\hline Optimal ${ }^{\ddagger}$ & 1.0 & $<.001$ & 1.0 & $<.001$ \\
\hline Adequate & $4.22(2.90,6.14)$ & & $3.52(2.40,5.17)$ & \\
\hline Poor & $6.84(4.52,10.36)$ & & $4.80(3.13,7.34)$ & \\
\hline \multicolumn{5}{|l|}{ Acquisition heart rate } \\
\hline$<60$ beats $/ \mathrm{min}^{\ddagger}$ & 1.0 & $<.001$ & 1.0 & .30 \\
\hline $60-70$ beats/min & $1.09(0.74,1.62)$ & & $0.93(0.65,1.32)$ & \\
\hline$>70$ beats/min & $2.83(2.10,3.81)$ & & $1.20(0.85,1.66)$ & \\
\hline
\end{tabular}

Note.-Data in parentheses are $95 \%$ Cls.

* Includes sex, age, segment calcium, segment image quality, acquisition heart rate, and the interaction of age and coronary calcium score. Standard error was adjusted for segments being clustered among 371 participants.

${ }^{\dagger}$ Mild $=<90^{\circ}$, moderate $=90^{\circ}-180^{\circ}$, severe $=>180^{\circ}$.

‡ Reference value.

calcium and lumen. Wall calcification and positive remodeling of the coronary vessel (external expansion of the vessel to accommodate plaque resulting in a reserved lumen) can be visualized in cross-sectional views with multidetector CT angiography. As a result, multidetector CT angiography shows more plaque and calcium than can be appreciated at CCA. In fact, CCA is limited to "lumenography" and is unable to show the extent of wall plaque and deep calcium. Therefore, multidetector CT angiography can show plaque that may not effect lumen dimensions. This is compounded in the presence of calcification in the wall, aforementioned artifacts, causing the plaque to appear to be encroaching on the lumen when it may not at CCA.

Technologic development to reduce blooming and beam-hardening artifacts has focused on improvements in reconstruction algorithms and spatial resolution. One approach has focused on the development of reconstruction algorithms to address beam-hardening artifacts and maximize spatial resolution (24). Other technical approaches to dense calcification include fast-switching dual-voltage technology and high-definition CT to improve spatial resolution and reduce artifacts $(25,26)$.

To our knowledge, this is the first 64-detector row study to implement a comprehensive coronary model (29segment coronary tree condensed to 19 segments) and to quantitatively measure the degree of luminal narrowing in each of the segments with both CCA and multidetector CT angiography. Another important strength of our study is its multicenter prospective nature and the thorough analysis of CCA and multidetector CT angiography studies in blinded centralized core laboratories. The clinical utility of multidetector CT angiography to identify a patient with obstructive CAD has been shown by CORE-64 and other studies (2-9); however, its utility to determine the presence or absence of substantial CAD in a segment is limited by the presence and extent of coronary artery calcification.

Our study was not without limitations. The qualitative assessment of coronary plaque and image quality may be influenced by subjectivity and interpretation bias. Additionally, individuals with a resting heart rate above 80 beats per minute, a body mass index greater than $40 \mathrm{~kg} / \mathrm{m}^{2}$, segments with stents, or segments that were less than $1.5 \mathrm{~mm}$ in diameter were excluded from our analysis.

In a symptomatic patient population, segment-based coronary artery calcification significantly decreased agreement between multidetector CT angiography and QCA for detection of a coronary stenosis of at least $50 \%$. Coronary calcification reduced diagnostic accuracy when controlling for sex, age, heart rate during image acquisition, and image quality. The prevalence of CAD in noncalcified segments was substantially lower than that in segments with mild, moderate, or severe calcification. In a segmentbased analysis, the positive likelihood ratio in a coronary segment with disease was acceptable in noncalcified segments, but was lower than is acceptable in segments with moderate or severe calcification. Negative likelihood ratios for coronary segments are limited. Importantly, there were differences in the diagnostic performance of multidetector CT angiography based on AUCs between noncalcified segments and mildly, moderately, and severely calcified segments. Further technologic development is needed to improve the assessment of lesion diameter stenosis in the setting of coronary calcification.

Disclosures of Potential Conflicts of Interest: A.L.V. Financial activities related to the present article: institution has a grant and receives support for travel to meetings from Toshiba Medical Systems. Financial activities not related to the present article: institution has a grant from Toshiba Medical Systems. Other relationships: none to disclose. A.A. Financial activities related to the present article: none to disclose. Financial activities not related to the present article: none to disclose. Other relationships: institution has a grant from Toshiba Medical Systems on behalf of the principal investigator of this study, for which A.A. is a member of the steering committee. C.E.R. Financial activities related to the pres ent article: receives support for travel to CORE 64 investigator meetings from Toshiba Medical Systems. Financial activities not related to the present article: none to disclose. Other relation ships: none to disclose. M.D. Financial activities related to the present article: institution has a grant from Toshiba Medical Systems. Financial activities not related to the present article: is a consultant for Guerbet; institution has grants from European Regional Development Fund, 
German Heart Foundation/German Foundation of Heart Research, GE Healthcare, Bracco, Guerbet, Toshiba Medical Systems, German Science Foundation (DFG), and German Federal Ministry of Education and Research (BMBF); is on the speakers bureau of Toshiba Medical Systems, Bayer-Schering, Guerbet, and Cardiac MR Academy Berlin; institution receives travel/accommodations expenses from Toshiba Medical Systems and Guerbet; is the course director for Cardiac CT Hands-on Courses at Charité in Berlin; institution has master research agreements with Siemens Medical Solutions, Philips Medical Systems, and Toshiba Medical Systems; receives compensation for book authorship from Springer. Other relationships: none to disclose. H.N. No potential conflicts of interest to disclose. I.G. No potential conflicts of interest to disclose. M.E.C. Financial activities related to the present article: none to disclose. Financial activities not related to the present article: institution has an $\mathrm{NIH} /$ NHLBI grant (no. 5P50HL083813-04). Other relationships: none to disclose. D.E.B. No potential conflicts of interest to disclose. J.W.M.H. Financial activities related to the present article: institution has a grant from Toshiba Medical Systems, receives support for travel to project committee meetings from Toshiba Medical Systems. Financial activities not related to the present article: institution has a grant from Toshiba Medical Systems, institution receives compensation for service on the speakers bureaus of Toshiba Medical Systems and Bayer Schering Pharma. Other relationships: none to disclose. A.d.R. Financial activities related to the present article: institution has a grant from Toshiba. Financial activities not related to the present article: individual and institution receive compensation for second opinion testimony from an insurance company, institution receives compensation for service on the speakers bureau of Guerbet. Other relationships: none to disclose. C.C. Financial activities related to the present article: institution has a grant and receives support for travel to meetings from Toshiba Medical Systems. Financial activities not related to the present article: institution has a grant from Toshiba Medical Systems. Other relationships: none to disclose. No potential conflicts of interest to disclose. J.A.C.L. Financial activities related to the present article: institution has a grant from Toshiba Medical Systems, institution receives support for travel to project meetings from Toshiba Medical Systems. Financial activities not related to the present article: institution has a grant. Other relationships: none to disclose. J.M.M. Financial activities related to the present article: institution has grants from Toshiba Medical Systems and Doris Duke Charitable Foundation, individual and institution receive honoraria from Toshiba Medical Systems, institution receives support for travel to study meetings and educational purposes from Toshiba Medical Systems. Financial activities not related to the present article: none to disclose. Other relationships: none to disclose.

\section{References}

1. Lloyd-Jones D, Adams R, Carnethon M, et al. Heart disease and stroke statistics: 2009 update-a report from the American Heart
Association Statistics Committee and Stroke Statistics Subcommittee. Circulation 2009; 119(3):e21-e181.

2. Brodoefel H, Burgstahler C, Tsiflikas I, et al. Dual-source CT: effect of heart rate, heart rate variability, and calcification on image quality and diagnostic accuracy. Radiology $2008 ; 247(2): 346-355$.

3. Cordeiro MA, Miller JM, Schmidt A, et al. Non-invasive half millimetre 32 detector row computed tomography angiography accurately excludes significant stenoses in patients with advanced coronary artery disease and high calcium scores. Heart 2006;92(5):589-597.

4. Hamon M, Morello R, Riddell JW, Hamon M. Coronary arteries: diagnostic performance of 16- versus 64-section spiral CT compared with invasive coronary angiography - metaanalysis. Radiology 2007;245(3):720-731.

5. Meijboom WB, Meijs MF, Schuijf JD, et al. Diagnostic accuracy of 64-slice computed tomography coronary angiography: a prospective, multicenter, multivendor study. J Am Coll Cardiol 2008;52(25):2135-2144.

6. Miller JM, Rochitte CE, Dewey M, et al. Diagnostic performance of coronary angiography by 64 -row CT. N Engl J Med 2008 359(22):2324-2336.

7. Raff GL, Gallagher MJ, O'Neill WW, Goldstein JA. Diagnostic accuracy of noninvasive coronary angiography using 64 -slice spiral computed tomography. J Am Coll Cardiol 2005;46(3):552-557.

8. Dewey M, Vavere AL, Arbab-Zadeh A, et al. Patient characteristics as predictors of image quality and diagnostic accuracy of MDCT compared with conventional coronary angiography for detecting coronary artery stenoses: CORE-64 multicenter international trial. AJR Am J Roentgenol 2010;194(1):93-102.

9. Budoff MJ, Dowe D, Jollis JG, et al. Diagnostic performance of 64-multidetector row coronary computed tomographic angiography for evaluation of coronary artery stenosis in individuals without known coronary artery disease: results from the prospective multicenter ACCURACY (Assessment by Coronary Computed Tomographic Angiography of Individuals Undergoing Invasive Coronary Angiography) trial. J Am Coll Cardiol 2008;52(21):1724-1732.

10. Dewey M, Hamm B. CT coronary angiography: examination technique, clinical results, and outlook on future developments [in German]. Rofo 2007;179(3):246-260.

11. Hausleiter J, Meyer T, Hadamitzky M, et al. Non-invasive coronary computed tomographic angiography for patients with suspected coronary artery disease: the Coronary Angiography by Computed Tomography with the Use of a Submillimeter Resolution (CACTUS) trial. Eur Heart J 2007;28(24):3034-3041.

12. Pugliese F, Mollet NR, Hunink MG, et al. Diagnostic performance of coronary CT angiography by using different generations of multisection scanners: single-center experience. Radiology 2008;246(2):384-393.
13. Ong TK, Chin SP, Liew CK, et al. Accuracy of 64-row multidetector computed tomography in detecting coronary artery disease in 134 symptomatic patients: influence of calcification. Am Heart J 2006;151(6):1323.e1-1323.e6.

14. Meng L, Cui L, Cheng Y, et al. Effect of heart rate and coronary calcification on the diagnostic accuracy of the dual-source CT coronary angiography in patients with suspected coronary artery disease. Korean J Radiol 2009;10(4):347-354.

15. Gottlieb I, Miller JM, Arbab-Zadeh A, et al. The absence of coronary calcification does not exclude obstructive coronary artery disease or the need for revascularization in patients referred for conventional coronary angiography. J Am Coll Cardiol 2010;55(7):627-634.

16. Miller JM, Dewey M, Vavere AL, et al. Coronary CT angiography using 64 detector rows: methods and design of the multi-centre tria CORE-64. Eur Radiol 2009;19(4):816-828.

17. Dewey M, Laule M, Krug L, et al. Multisegment and halfscan reconstruction of 16 slice computed tomography for detection of coronary artery stenoses. Invest Radiol 2004; 39(4):223-229

18. Mintz GS, Popma JJ, Pichard AD, et al Patterns of calcification in coronary artery disease: a statistical analysis of intravascular ultrasound and coronary angiography in 1155 lesions. Circulation 1995;91(7):1959-1965.

19. Anderberg M. Cluster analysis for applications. New York, NY: Academic Press, 1973; 161.

20. Zou KH, O’Malley AJ, Mauri L. Receiveroperating characteristic analysis for evaluating diagnostic tests and predictive models. Circulation 2007;115(5):654-657.

21. Efron B, Tibshirani RJ. An introduction to the bootstrap. London, England: Chapman \& Hall, 1993

22. Zhang S, Levin DC, Halpern EJ, Fischman D, Savage M, Walinsky P. Accuracy of MDCT in assessing the degree of stenosis caused by calcified coronary artery plaques. AJR Am J Roentgenol 2008;191(6):1676-1683.

23. Dewey M. Coronary CT versus MR angiog raphy: pro CT - the role of CT angiography. Radiology $2011 ; 258(2): 329-339$.

24. Kitagawa K, George RT, Arbab-Zadeh A, Lima JA, Lardo AC. Characterization and correction of beam-hardening artifacts during dynamic volume CT assessment of myocardial perfusion. Radiology 2010;256(1):111-118.

25. Yamada M, Jinzaki M, Imai Y, et al. Evaluation of severely calcified coronary artery using fast-switching dual-kVp 64-slice computed tomography. Circ J 2011;75(2):472-473.

26. Tanami Y, Jinzaki M, Yamada M, Imai Y Segawa K, Kuribayashi S. Improvement of in-stent lumen measurement accuracy with new High-Definition CT in a phantom model: comparison with conventional 64-detector row CT. Int J Cardiovasc Imaging doi:10 1007/s10554-010-9786-x. Published online January 8, 2011. Accessed May 11, 2011. 